\title{
Evaluation of Cervico-occlusal Dimensions of Maxillary and Mandibular Incisor Brackets for Lingual Orthodontics
}

\author{
${ }^{1}$ Andréia Cotrim-Ferreira, ${ }^{2}$ Flávio Cotrim-Ferreira, ${ }^{3}$ Flávio Vellini-Ferreira, ${ }^{4}$ Daniele Fernanda Peron \\ ${ }^{5}$ Carlos Carvalho, ${ }^{6}$ Fernando César Torres
}

\section{ABSTRACT}

Aim: The purpose of this study was to analyze the cervicoocclusal dimensions of brackets used in lingual orthodontics for maxillary and mandibular central and lateral incisors, to compare them with the numbers informed by the manufacturer.

Materials and methods: The brackets were placed in a template and analyzed by scanning electron microscopy (SEM), evaluating the images with the aid of a software that allowed determination of reference points, tracing of lines on the bracket images and measurement of the distances.

Results: Brackets manufactured by Dm Ceosa Hiro, Tecnident PSWb and Dentaurum Magic did not present statistically significant differences between the mean values of slot heights and the values informed by manufacturers. Conversely, the brands Ormco 7th generation, Ormco STb, GAC Innovation L, ORJORG, Tecnident, Ormco STb LSW and Adenta evolution STL exhibited statistically significant differences.

Conclusion: In general, there was a deficiency in the standardization of cervico-occlusal measurements of slots of the brackets analyzed.

Clinical significance: Manufacturers should be attentive to the quality control of their materials. Future studies will analyze the actual clinical influence of these findings on the orthodontic mechanics.

Keywords: Braces, Torque, Orthodontics, Corrective, Laboratory research.

How to cite this article: Cotrim-Ferreira A, Cotrim-Ferreira F, Vellini-Ferreira F, Peron DF, Carvalho C, Torres FC. Evaluation of Cervico-occlusal Dimensions of Maxillary and Mandibular Incisor Brackets for Lingual Orthodontics. J Contemp Dent Pract 2014;15(3):306-311.

Source of support: Nil

Conflict of interest: None declared

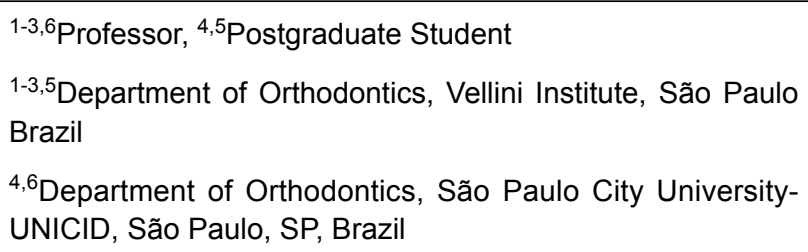

Corresponding Author: Fernando César Torres, Professor Department of Orthodontics, São Paulo City UniversityUNICID, São Paulo, Brazil, e-mail: fernandozzy@hotmail. com

\section{INTRODUCTION}

The torque in orthodontics may be defined as root displacement produced by torsion of the archwire, whose force is transmitted to the tooth by the bracket or tube. ${ }^{1}$ From a biomechanical standpoint, the adequate inclination of incisors during orthodontic treatment is important to achieve good occlusal outcomes, allowing achievement of the desired overjet, closure of extraction spaces and correct intercuspation.

The smile esthetics and lip support are also influenced by the torque of incisors. The torque was the last force introduced during the development of orthodontics, possibly being the most difficult to understand and achieve. It is so important that the edgewise technique received this name because of the use of rectangular archwires, which allowed the control of tooth inclination. ${ }^{2}$

The advent of preadjusted brackets was undoubtedly one of the great evolutions in orthodontics. ${ }^{3}$ As part of the concept of straight-wire appliances, the application of torque was less dependent on torsion of rectangular archwires around their long axis, because the torque was inserted in the base or slot of brackets and tubes, simplifying the orthodontic mechanics.

However, some authors observed that the treatment objectives are not always reached even when preadjusted appliances are used, requiring additional bends in the archwires. This would be related to failures in bonding, irregularities in tooth surfaces, abnormal crown and root angulations, and space between the bracket and the archwire, which may occur depending on the accessories and archwires selected. Other relevant aspect would be the manufacturer allowance in the standardization of orthodontic materials. A previous study ${ }^{4}$ demonstrated that 0.018 inch slots ranged from 0.0182 to 0.0192 inches. According to the authors, this would correspond to a space of $6^{\circ}$, between a $0.018 \times 0.025$ slot and a $0.018 \times 0.025$ archwire.

Knowledge on the accuracy of bracket slots is fundamental in orthodontics because it influences the correct force application. Manufacturers usually do not diffuse the measurement method employed, and most studies use digital pachymeters (Mitutoyo Corp, 516 series) with $0.01 \mathrm{~mm}$ accu- 
Table 1: Brand, slot size according to manufacturer, and country of origin

\begin{tabular}{lll}
\hline Brands & Slot & Origin \\
\hline Adenta evolution STL & $711 \mu \mathrm{m} / 0.028 "$ & USA \\
Dentaurum Magic & $510 \mu \mathrm{m} / 0.020 "$ & Germany \\
Dm Ceosa Hiro & $457 \mu \mathrm{m} / 0.018 "$ & Spain \\
Ormco 7gen & $457 \mu \mathrm{m} / 0.018 "$ & USA \\
Ormco STb & $457 \mu \mathrm{m} / 0.018 "$ & USA \\
GAC In-Ovation L & $457 \mu \mathrm{m} / 0.018 "$ & USA \\
Tecnident PSWb & $457 \mu \mathrm{m} / 0.018 "$ & Brazil \\
ORJ-ORG & $457 \mu \mathrm{m} / 0.018 "$ & Japan \\
Tecnident & $457 \mu \mathrm{m} / 0.018 "$ & Brazil \\
Ormco STb LSW & $457 \mu \mathrm{m} / 0.018 "$ & USA \\
\hline
\end{tabular}

racy. Kusy and Whitley, ${ }^{5}$ in 1999 , examined 24 brackets from eight manufacturers and found three slots with smaller measurements than indicated, while 20 presented greater dimensions than indicated by the manufacturers. The greatest slot found in $0.018^{\prime}$ brackets was $6 \%$ greater than declared, while the greatest slots in $0.022^{\prime}$ brackets were $8 \%$ greater.

Due to the increasing esthetic demands of patients, lingual orthodontics has been disseminated and enhanced. Since, the onset of its development to the present date, several lingual brackets have been designed and modified, and new laboratory procedures have been incorporated to enhance the outcomes, increase the mechanical efficiency and the patient comfort.

Similar to conventional brackets, studies should be conducted to evaluate the quality and accuracy of lingual brackets, which may influence the mechanics and consequently the orthodontic treatment outcomes. Therefore, this study compared, by scanning electron microscopy (SEM), the cervico-occlusal dimensions of lingual brackets of incisors, compared to their expected dimensions according to the manufacturers and comparing the different brands according to their accuracy.

\section{MATERIALS AND METHODS}

A total of 80 lingual brackets of maxillary and mandibular central and lateral incisors were analyzed concerning the size of their slots, defining this distance as the cervico-occlusal dimension. Ten brands were analyzed, including eight brackets (one of each incisor) of each following brand: Adenta, GAC, ORJ-ORG, Ormco Stb, Ormco Stb LSW, Ormco 7th generation, Tecnident, Tecnident Prieto, Dentaurum, Dm Ceosa. The bracket brands, slot sizes according to the manufacturers, and country of origin are presented in Table 1.

The following materials were used: 16 rectangular aluminum bars with accurate straight angles, measuring $5 \times 15 \times$ $30 \mathrm{~mm}$ (Fig. 1A); cyanoacrylate adhesive, brand Super Bonder; wooden base measuring $19 \times 20 \mathrm{~cm}$; millimeter paper; rectangular stainless steel $0.017 " \times 0.025$ " wire; SEM, model Philips XL-30; software Microscope Control. The millimeter paper was trimmed and bonded in each wooden base. Thereafter, the aluminum bars were positioned and bonded, so as they were adjusted parallel and perpendicular to the millimeter paper (Fig. 1B).

Five brackets were positioned on each aluminum base, adding up to 16 aluminum bars. A segment of rectangular stainless steel $0.017^{\prime \prime} \times 0.025^{\prime \prime}$ wire, perfectly straight from the manufacturer, was used as a guide to bond each bracket. The wire was positioned coincident with the millimeter paper and perpendicular to the long axis of the aluminum bar (Fig. 1C), so as the brackets were bonded in a standardized manner and their slots were observed in lateral view, as displayed in Figure 2.

The brackets were bonded with cyanoacrylate adhesive (Super Bonder), which was selected to maintain a dry surface, which was necessary for achievement of images on the SEM. ${ }^{6}$ On the SEM, the focus was adjusted in each bracket (Fig. 3),
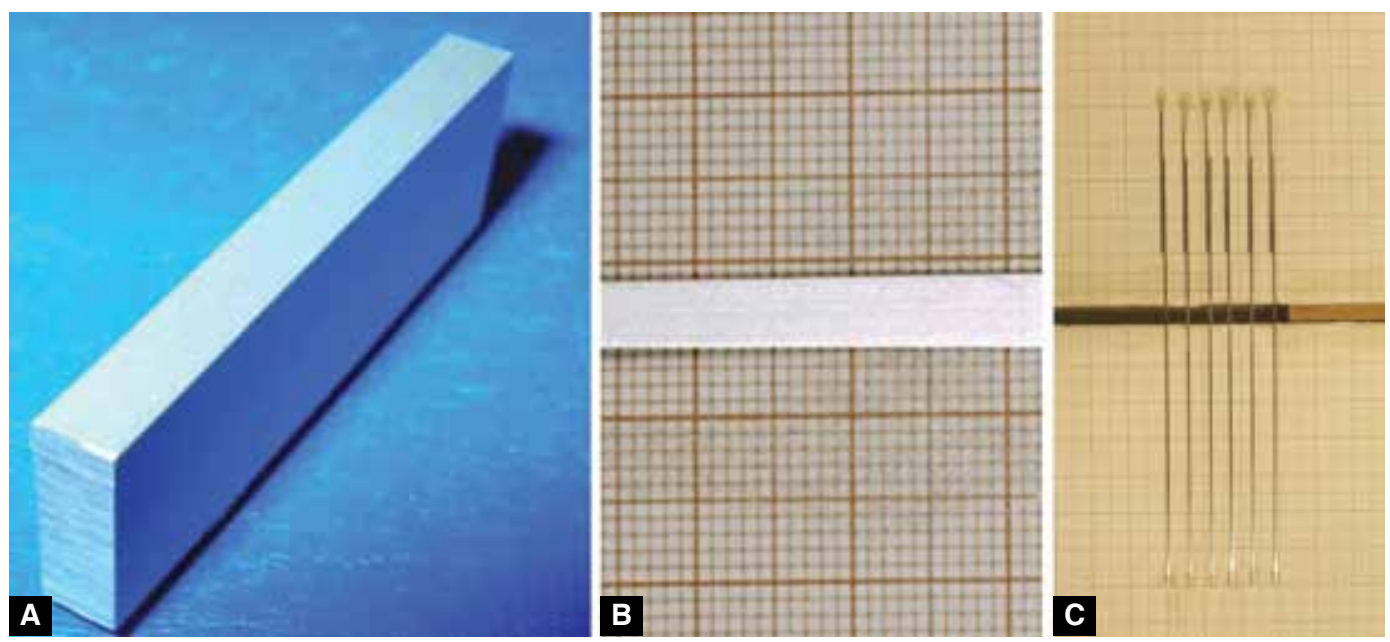

Figs 1A to C: Aluminum bar employed $(A)$, positioning of the bar on the wooden base with bonded millimeter paper (B) and archwires used as a guide for bonding of brackets (C) 


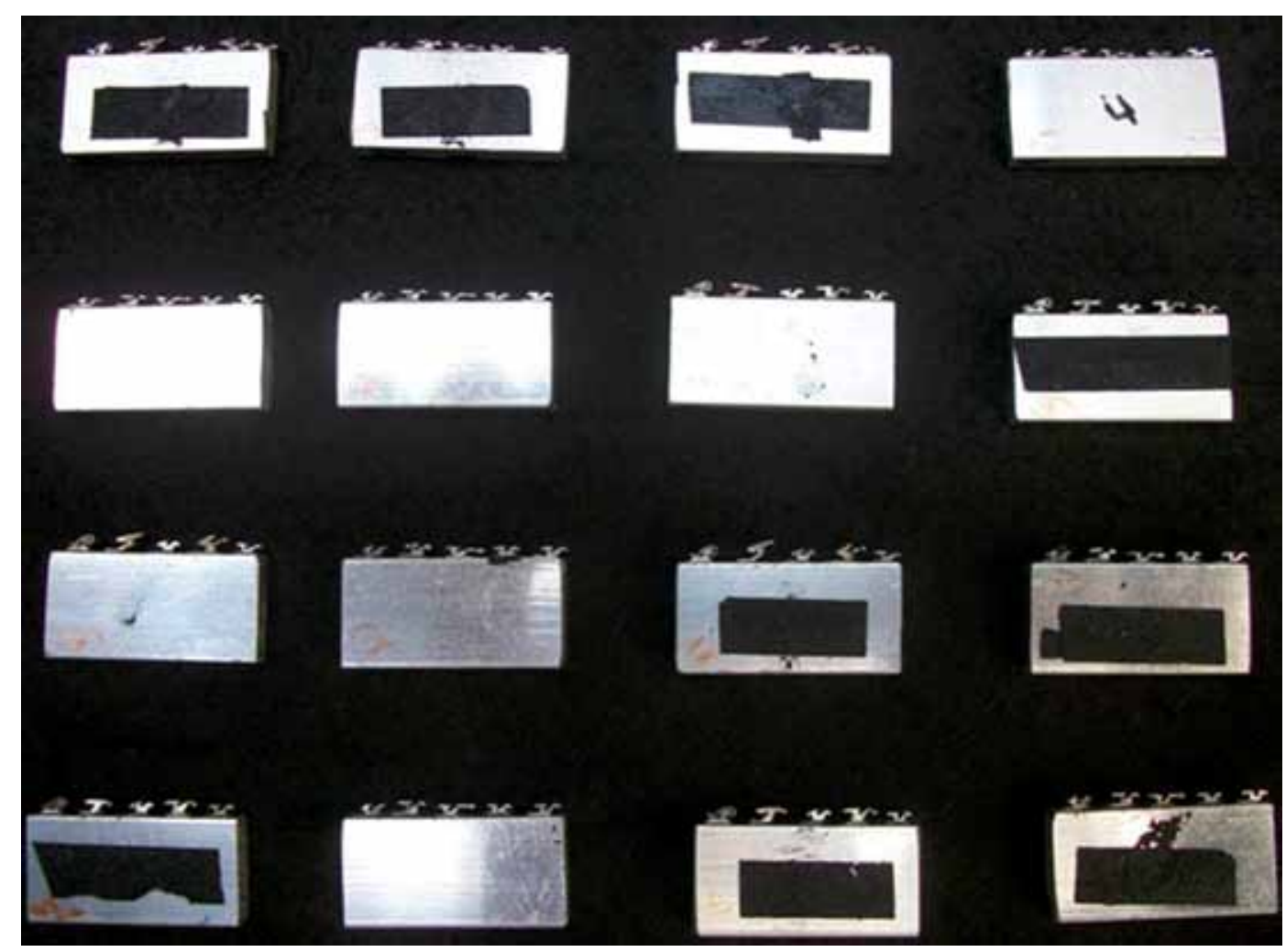

Fig. 2: Lateral visualization of bonded brackets

so that the profile was as clear as possible, with $45 \times$ magnification throughout its extension. After achievement, the images were stored on the microscope computer, processed and measured.

Measurements were performed on points obtained from the intersection of lines representing the cervical and incisal walls of brackets and other on the bottom of each slot. The reference points A and B were then defined respectively, representing the incisal and cervical bracket walls, i.e. the cervico-occlusal dimension to be analyzed (Fig. 4). Points were used on the bottom of slots because rectangular and/ or square archwires transfer the incorporated torques at this region. Figure 4 further demonstrates the measurement performed on the same software used for observation of SEM images.

\section{RESULTS}

For evaluation of the cervico-occlusal dimension of slots, this study presents a dependent variable, the "fitting dimension' (FD), measured in micrometers $(\mu \mathrm{m})$ and the variation factor are the 'brands'. Data were compared by analysis of variance (ANOVA). For that purpose, the normality and homogeneity were tested by the Shapiro-Wilk and Levene tests respectively. The Shapiro-Wilk (SW) test indicated that the variable 'FD' presented normal distribution, with values of $p>0.05$. The Levene test demonstrated that the variable 'FD' did not present homogeneity $(\mathrm{p}<0.05)$, with very different confidence intervals of standard deviations.
The result of the ANOVA test was significant, with $p$ $<0.05$. Therefore, there was evidence to accept the hypothesis of statistically significant influence of the Brand on the cervico-occlusal fitting dimension.

The descriptive statistics is presented in Table 2 and the results may also be observed in Figure 5. To compare the real measurements and those indicated by the manufacturer, each brand was compared to a fixed value (informed by the manufacturer) by the one-sample t-test (Table 3).

\section{DISCUSSION}

This study aimed to contribute to enhance the lingual brackets, which have been increasingly used in the orthodontic practice. The SEM demonstrated to be a very effective technique for visualization and measurement of bracket slots, with advantages compared to evaluations performed by light microscopy. The SEM presents greater capacity to achieve images in depth, with optimal definition of relief and shape. ${ }^{6}$

Among all brands with values of $457 \mu \mathrm{m}$ or 0.018 ' tested', the brands Dm Ceosa Hiro and Tecnident PSWb presented lower calculated t-values than the critical value of 2.36 , thus nonsignificant $(p>0.05)$. These brands were not statistically different from the value informed by the manufacturer (reference value of $457 \mu \mathrm{m}$ or $0.018 "$ ). For both brands, the reference value is within the observed values, being that 95\% confidence intervals for both include the zero. Graphically, it may be observed that the dotted ref line intercepts the confidence intervals of the aforementioned brands. 


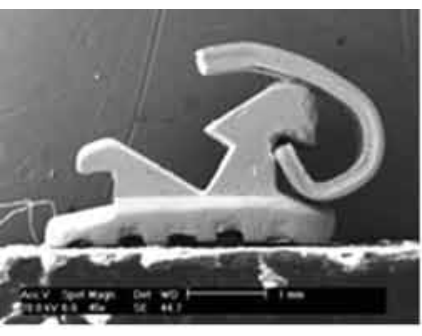

Adenta

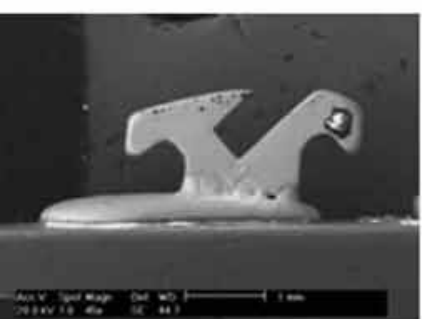

Dm Ceosa

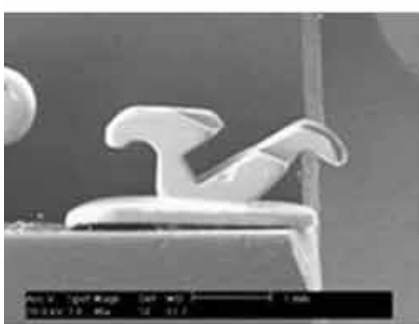

Ormco STb

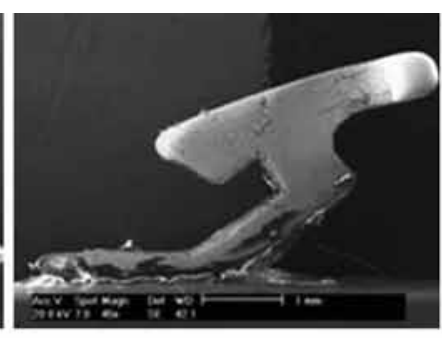

Dentaurum

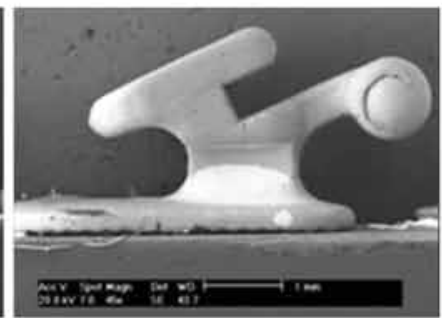

Ormco 7 gen

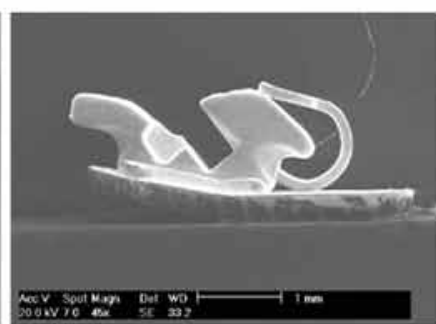

GAC

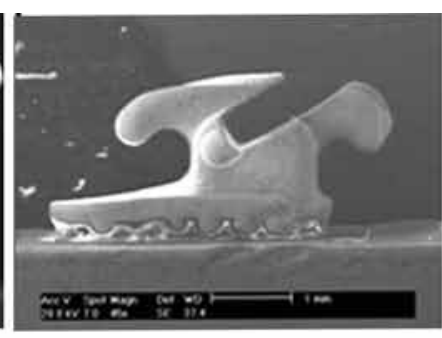

Tecnident PSWb

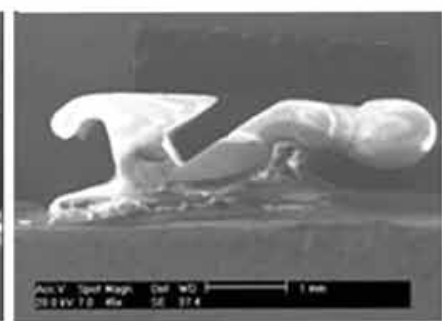

ORJ

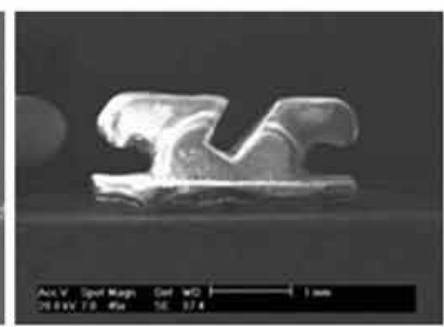

Tecnident

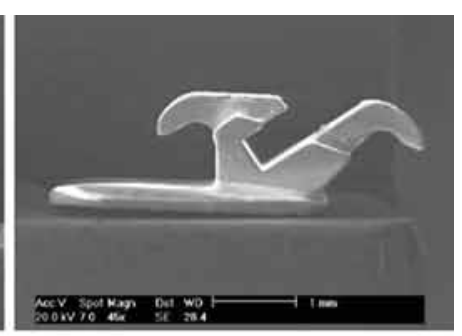

Ormco STb LSW

Fig. 3: Images of each bracket brand obtained on the SEM

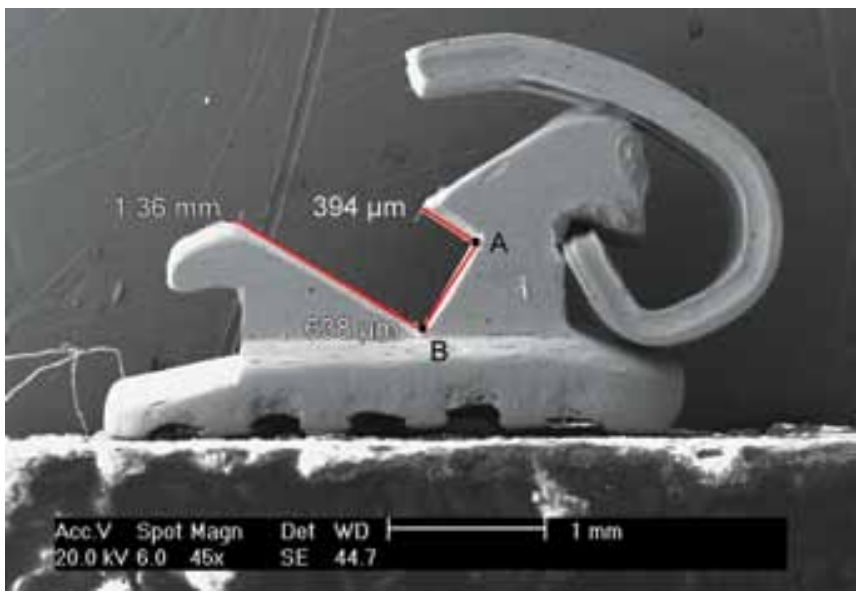

Fig. 4: Reference lines and points and measurement performed

It was observed that the brands Ormco 7th generation, Ormco STb, GAC inovation L, ORJ-ORG, Tecnident and Ormco STb LSW presented higher $\mathrm{t}$ values than the critical value of 2.36 and thus were significant $(p<0.05)$. These brands were statistically different from the value informed by the manufacturer (reference value of $457 \mu \mathrm{m}$ or 0.018 "). For these brands, the reference value is not within the observed values and the $95 \%$ confidence intervals do not include the zero. Among the aforementioned brands, the brand Ormco 7th generation presented statistically higher value [above the dotted line (Fig. 5)] and the brands GAC inovation L, ORJ-ORG, Ormco STb, Ormco STb LSW and Tecnident exhibited statistically

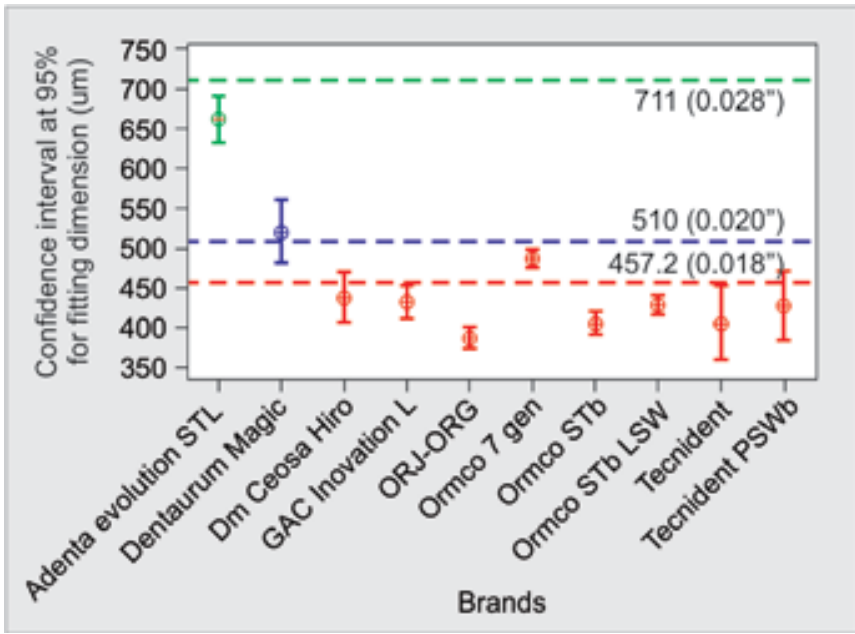

Fig. 5: Ninety-five percent confidence intervals in comparisons between values observed and the reference values represented by dotted lines

lower values in relation to reference values [below the dotted line (see Fig. 5)].

The blue dotted line represents the reference value of $0.510 \mu \mathrm{m}(0.020 ")$, only for the brand Dentaurum Magic $(t=0.75 ; p=0.48)$. For this brand, it was observed that the calculated t-value was lower than the critical value of 2.36 and thus was not significant ( $p>0.05$ ), being that the H0 was not rejected, i.e. there was no statistically significant difference from the value informed by the manufacturer (reference value of $510 \mu \mathrm{m}$ or $0.020 "$ ). The reference value 
Table 2: Statistical parameters and descriptive statistics of experimental data (unit $=\mu \mathrm{m})$

\begin{tabular}{|c|c|c|c|c|c|c|c|c|c|}
\hline Brands & $N$ & Mean $(\mu m)$ & $S D$ & $S E$ & $\begin{array}{l}\text { Lower limit } \\
(95 \%)\end{array}$ & $\begin{array}{l}\text { Upper limit } \\
(95 \%)\end{array}$ & Min. & Max. & CV (\%) \\
\hline Adenta evolution STL & 8 & 661.75 & 33.72 & 11.92 & 633.56 & 689.94 & 613 & 706 & 5.10 \\
\hline Dentaurum Magic & 8 & 522.25 & 46.23 & 16.34 & 483.6 & 560.9 & 448 & 606 & 8.85 \\
\hline Dm Ceosa Hiro & 8 & 438.13 & 38.2 & 13.51 & 406.19 & 470.06 & 389 & 495 & 8.72 \\
\hline Ormco 7 gen & 8 & 486.63 & 13.55 & 4.79 & 475.29 & 497.96 & 465 & 503 & 2.78 \\
\hline Ormco STb & 8 & 405.88 & 16.32 & 5.77 & 392.23 & 419.52 & 383 & 433 & 4.02 \\
\hline GAC Inovation L & 8 & 432.38 & 23.41 & 8.28 & 412.8 & 451.95 & 402 & 472 & 5.41 \\
\hline Tecnident PSWb & 8 & 427 & 50.98 & 18.02 & 384.38 & 469.62 & 354 & 489 & 11.94 \\
\hline ORJ-ORG & 8 & 388.25 & 16.51 & 5.84 & 374.45 & 402.05 & 367 & 421 & 4.25 \\
\hline Tecnident & 8 & 405.75 & 55.19 & 19.51 & 359.61 & 451.89 & 319 & 470 & 13.60 \\
\hline Ormco STb LSW & 8 & 429.38 & 13.81 & 4.88 & 417.83 & 440.92 & 412 & 457 & 3.22 \\
\hline Total & 80 & 459.73 & 84.15 & 9.4 & 441.01 & 478.46 & 319 & 706 & 18.30 \\
\hline
\end{tabular}

SD: Standard deviation; SE: Standard error; Lower limit (95\%): Lower limit of the confidence interval at 95\%; Upper limit (95\%): Upper limit of the confidence interval at 95\%; Min: Minimum; Max: Maximum; CV (\%): Coefficient of variation

Table 3: One-sample t-test compared to the value informed by the manufacturer (reference value) for all brands

\begin{tabular}{lrllllr}
\hline Brands & $t$-value & $d f$ & Sig $(p)$. bicaudal & Mean difference $(\mu \mathrm{m})$ & Lower limit (95\%) & Upper limit (95\%) \\
\hline Adenta evolution STL & -4.13 & 7 & $4.00 \mathrm{E}-03^{*}$ & -49.25 & -77.44 & -21.06 \\
Dm Ceosa Hiro $^{\dagger}$ & -1.40 & 7 & 0.20 & -18.88 & -50.81 & 13.06 \\
${\text { Ormco } 7 \text { gen }^{\dagger}}$ & 6.18 & 7 & $4.53 \mathrm{E}-04^{*}$ & 29.63 & 18.29 & 40.96 \\
Ormco STb $^{\dagger}$ & -8.86 & 7 & $4.73 \mathrm{E}-05^{*}$ & -51.13 & -64.77 & -37.48 \\
GAC Inovation L $^{\dagger}$ & -2.98 & 7 & $0.02^{*}$ & -24.63 & -44.20 & -5.05 \\
Tecnident PSWb $^{\dagger}$ & -1.66 & 7 & 0.14 & -30.00 & -72.62 & 12.62 \\
ORJ-ORG $^{\dagger}$ & -11.78 & 7 & $7.20 \mathrm{E}-06^{*}$ & -68.75 & -82.55 & -54.95 \\
Tecnident $^{\dagger}$ & -2.63 & 7 & $0.03^{*}$ & -51.25 & -97.39 & -5.11 \\
Ormco STb LSW $^{\dagger}$ & -5.66 & 7 & $7.70 \mathrm{E}-04^{*}$ & -27.63 & -39.17 & -16.08 \\
Dentaurum Magic $^{\ddagger}$ & 0.75 & 7 & 0.48 & 12.25 & -26.40 & 50.90
\end{tabular}

Experimental unit ( $\mu \mathrm{m}) ;{ }^{\phi}$ Reference value: 0.711 (0.028"); ${ }^{\dagger}$ Reference value: 0.457 (0.018"); ${ }^{\ddagger}$ Reference value: 0.510 (0.020"); Critical t-value $(0.05 ; 7):|2.36| ;{ }^{*} p<0.05$

is within the observed values, and the confidence interval includes the zero. Graphically, it may be observed that the blue dotted line intercepts the confidence interval.

The green dotted line represents the reference value of $711 \mu \mathrm{m}(0.028 ")$, only for the brand Adenta evolution STL. For this brand, it was observed that the calculated t-value was higher than the critical value of 2.36 and thus significant $(\mathrm{p}<0.05)$, therefore, the H0 was rejected and the H1 was accepted, i.e. there is statistically significant difference from the value informed by the manufacturer (reference value of $711 \mu \mathrm{m}$ or $0.028 ")$. The reference value is not within the observed values, and the confidence interval does not include the zero. The brand Adenta presented statistically lower values than the reference value, graphically below the dotted line, which does not intercept the confidence interval.

Even though this was not the study objective, it was observed that the walls of slots analyzed presented irregularities, i.e. in some brackets they were not parallel to each other as they should be. This divergence or convergence might further increase the inaccuracy of brackets, ${ }^{7-9}$ highlighting the difficulty in the standardization and quality control in the manufacturing process of these orthodontic braces.
This finding evidences that manufacturers should be attentive to the quality control of their materials, combined to the main objective of this study, namely to verify the slot height of different lingual brackets. The results indicated that there is large variation in the cervico-occlusal dimension of lingual brackets analyzed, with more or less significant discrepancies. In general, the brackets analyzed presented smaller dimensions than those mentioned in their respective brands, and some brands did not present the respective dimensions stated by the manufacturers. One brand exhibited higher values than the informed. Future studies should analyze the actual clinical influence of these findings on the orthodontic mechanics.

\section{CONCLUSION}

According to the present methodology, the results indicated that:

- Brackets of brands Dm Ceosa Hiro, Tecnident PSWb and Dentaurum Magic did not present statistically significant differences between the mean values of slot heights and the values informed by manufacturers. 
- The brands Ormco 7th generation, Ormco STb, GAC inovation L, ORJ-ORG, Tecnident, Ormco STb LSW and Adenta evolution STL exhibited statistically significant differences $(p<0.05)$ in relation to the mean value of slot height, informed by the manufacturers. The brand Ormco 7th generation exhibited statistically higher value, while the other brands presented statistically smaller values compared to the informed reference values.

- In general, there was deficiency in the standardization of cervico-occlusal dimensions of bracket slots for each brand analyzed.

\section{REFERENCES}

1. Odegaard J, Meling T, Meling E. An evaluation of torsional moments developed in orthodontics applications. Am J Orthod 1994;105(4):392-400.
2. Angle EH. Evolution of orthodontia: recent developments. Dental Cosmos, Philadelphia, 1929;71(3):260-270.

3. Andrews LF. The six keys to normal occlusion. Am J Orthod 1972;62(3):296-309.

4. Creekmore T, Kunik RL. Straight-wire: the next generation. Am J Orthod Dentofacial Orthop 1993;104(1):8-20.

5. Kusy RP, Whitley JQ. Assessment of second-order clearances between orthodontic archwire and bracket slots via the critical contact angle for binding. Angle Orthod 1999;69(1):71-80.

6. Maliska AM. Scanning electron microscopy. Florianópolis, Brazil: UFSC 2006.

7. Sebanc J, Brantley W, Pincsak JJ, Conover JP. Variability of effective root torque as a function of edge bevel on orthodontic and wires. Am J Orthod 1984;86(1):43-51.

8. Kapur-Wadhwa R, Sinhá PK, Nanda RS. Comparison of load transmission and bracket deformation between titanium and stainless steel brackets. Am J Orthod Dentofacial Orthop 1999;116(3):275-279.

9. Kapur-Wadhwa R. Physical and mechanical properties affecting torque control. J Clin Orthod 2004;38(6):335-340. 